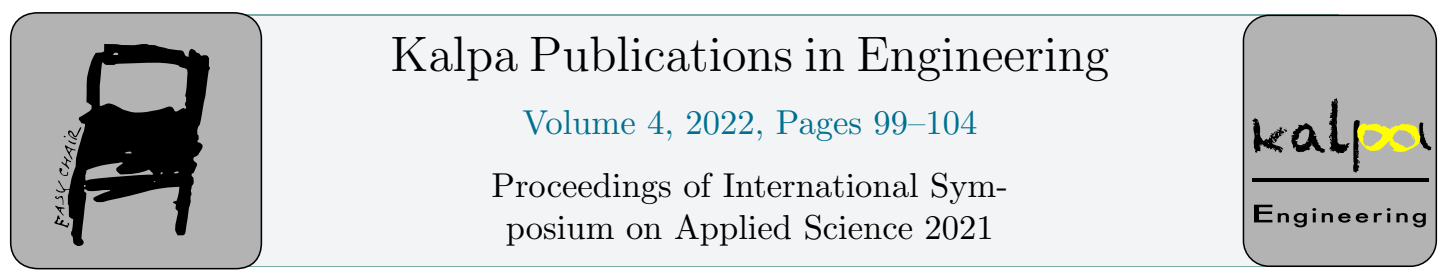

\title{
Study On Architectural Models Designed For Natural Ventilation By Finite Volume Method
}

\author{
Tich-Thien Truong ${ }^{1,2 *}$, Quoc-Thien Pham ${ }^{1,2}$, Kim-Bang Tran ${ }^{1,2}$ \\ ${ }^{1}$ Department of Engineering Mechanics, Faculty of Applied Science, Ho Chi Minh City \\ University of Technology (HCMUT), 268 Ly Thuong Kiet Street, District 10, Ho Chi Minh City, \\ Vietnam \\ ${ }^{2}$ Vietnam National University Ho Chi Minh City, Linh Trung Ward, Thu Duc District, Ho Chi \\ Minh City,Vietnam \\ tttruong@hcmut.edu.vn
}

\begin{abstract}
With people's health status according to statistics getting worse and worse, improving the quality of health is an inevitable need that many researchers are interested in. In addition to improving through eating, improving the living environment in homes and workplaces is also essential. Nowadays, many countries around the world have implemented many house models that apply natural ventilation instead of artificial air conditioning system, because natural wind is better and also feels more comfortable. Therefore, the study of controlled natural wind-catching architecture is necessary and consistent. Research in this field can help improve the living environment for people. The objective of the paper is to simulate ventilation solutions based on experience in construction works by finite volume method through ANSYS software to consider and evaluate the feasibility of these solutions. If the simulation results match or approximate the actual verified results, they can be applied to the improvement of natural ventilation structures to create a better indoor living environment, meeting the requirements of the environment. more comfortable diagnostics.
\end{abstract}

Keywords: Natural ventilation, finite volume method, ANSYS.

\section{Introduction}

Natural ventilation creates thermal comfort, higher humidity than mechanical ventilation systems. So now many places have researched smart home solutions to improve the quality of life. In which the design of controlled and automatic natural ventilation systems has a big role. Currently, there are no general principles to guide the design of automatic natural ventilation control systems in buildings. Meanwhile, the rapid development of smart homes with the requirement to automatically control technical systems, including the organization of natural ventilation, makes the study of control strategies 
difficult. Automatic ventilation becomes more and more necessary. The requirement for natural air intake to enhance indoor microclimate comfort requires the introduction of natural (pollution-free) air into the entire indoor usable area, and at the same time the velocity within the limits. $0.5-2.0 \mathrm{~m} / \mathrm{s}$ depending on the temperature and humidity of the outside air. The research objective is to simulate empirically based natural ventilation solutions using numerical methods to consider and evaluate the feasibility of these solutions, in order to improve natural ventilation architectures, meet Meet the criteria more comfortably.

\section{A Brief Of The Theory And Method}

\subsection{Turbulent flow}

The motion is chaotic and non-periodic, in which transmission quantities such as velocity, temperature, mass, etc. vary over time and space. The characteristic components of turbulent flow are turbulence eddies.

The properties of flow and velocity are representations of random values, results can only be obtained by enumerating the values and averaging.

The size of turbulence eddies has a wide range. Large turbulence eddies get energy from the flow, energy is transferred from large into small turbulence eddies. When the eddies are small enough, the turbulent energy is converted into the internal energy of the stream due to viscous dissipation.

\subsection{Computational methods for turbulence flow}

Direct Numerical Simulation (DNS): The DNS method solves the entire Navier-Stokes equation in full form and solves the large and small eddies in the turbulent stream without using modeling. The method has high accuracy but the computational cost is too large and cannot be applied to industry.

Large Eddy Simulation (LES): This method solves the Navier-Stokes equation only interested in spatially variable components. Only solve the equation at large vortices, vortices with a size smaller than the calculated grid will be modeled. LES still uses numerical methods to calculate, although the cost and resources needed are less than DNS, but it is still very high for practical problems.

Reynolds-Averaged Navier-Stokes (RANS): This method solves only the time-varying components of the Navier-Stokes equation. All large and small vortices and entangled components are modeled. The computational cost and resource usage of the RANS method are reasonable, so this method is widely applied in industry.

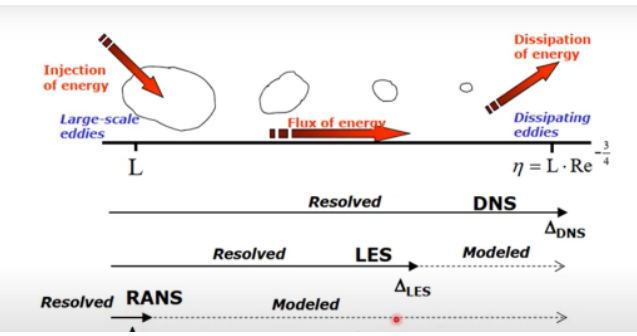

Figure 1: Comparison of turbulent current calculation methods

\subsection{Natural convection for turbulent flow}

In the response of turbulent flow, temperature is an unstable and variable variable, temperature is a function of the form: 


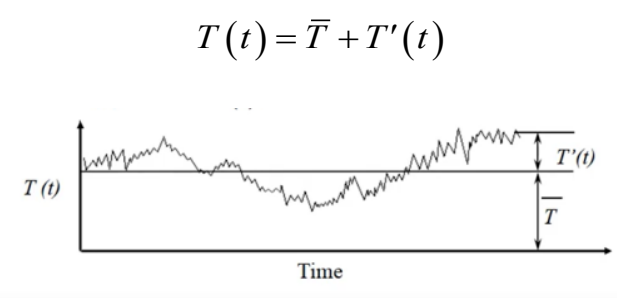

Figure 2: Graph of temperature function

Applying the RANS method, the energy equation has the following form

$$
\frac{\partial(\rho c \bar{T})}{\partial t}+\frac{\partial}{\partial x_{i}}(\rho c \bar{v} \bar{T})=\frac{\partial}{\partial x_{i}}(k \bar{T})-\frac{\partial}{\partial x_{i}}\left(\rho c \overline{v^{\prime} T^{\prime}}\right)(1)
$$

\section{Numerical Examples}

In simulation examples, natural ventilation solutions based on experience will be simulated. Thereby obtaining air velocity and temperature field results and comparing with comfort standards based on temperature and velocity.

To output temperature and velocity results on a cross-section, an elevation of about $1 \mathrm{~m} 6$ is selected.

The turbulent current model used will be the k-e model with the following advantages: ensuring a balance between computational costs and high accuracy; simple model, need little input data for calculation; can be used for a variety of industrial flows; It is well established and has been extensively tested for reliability.

The climate data are taken as follows: the average air temperature is 27 degrees Celsius and the average wind speed is $4.5 \mathrm{~m} / \mathrm{s}$.

\subsection{Problem 1}

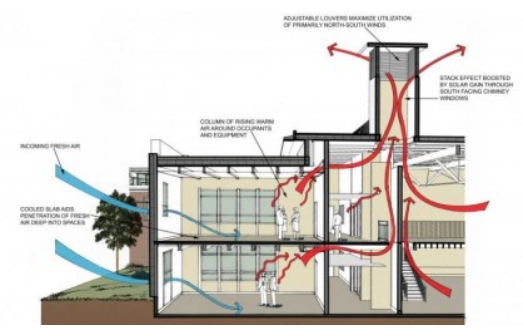

Figure 3: Natural ventilation solutions based on experience

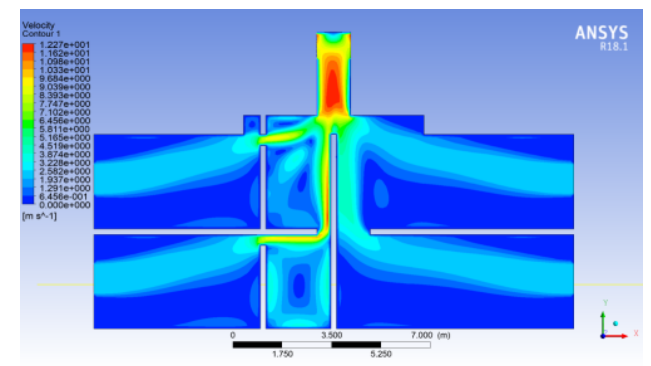

Figure 5: Velocity field 


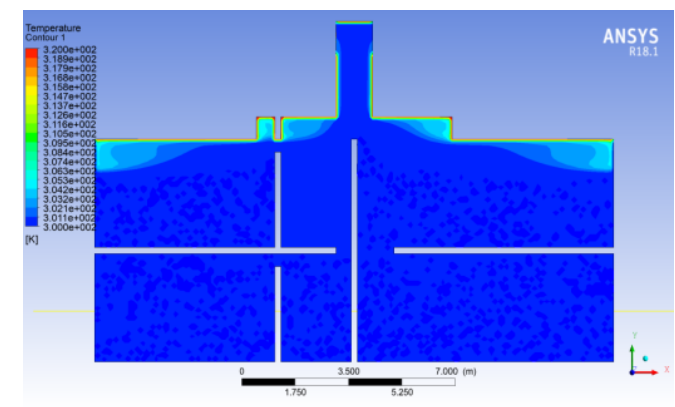

Figure 6: Temperature field

\subsection{Problem 2}

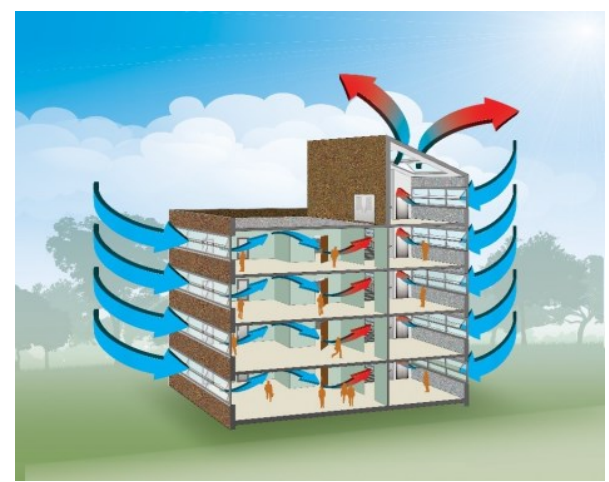

Figure 7: Natural ventilation solutions based on experience

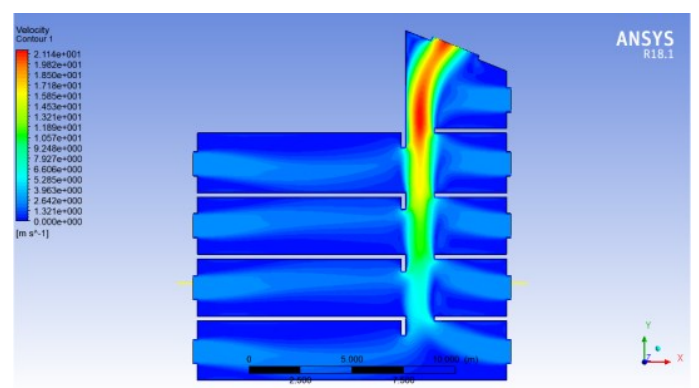

Figure 8: Velocity field

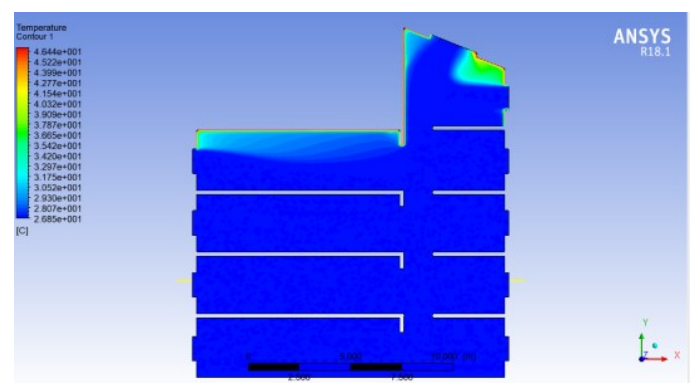

Figure 9: Temperature field 


\subsection{Problem 3}

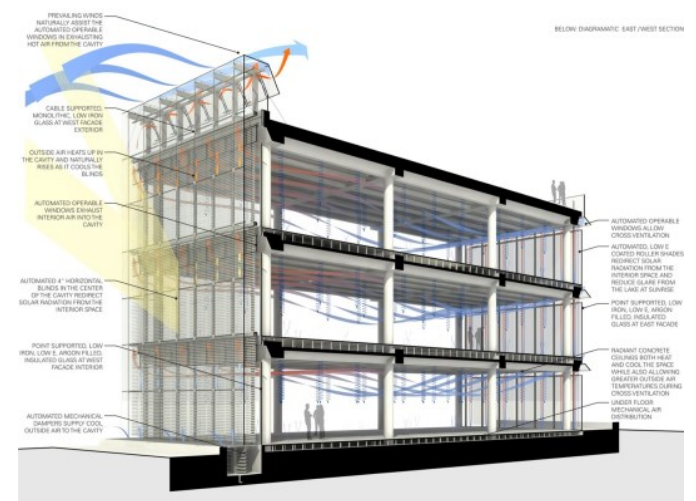

Figure 10: Natural ventilation solutions based on experience
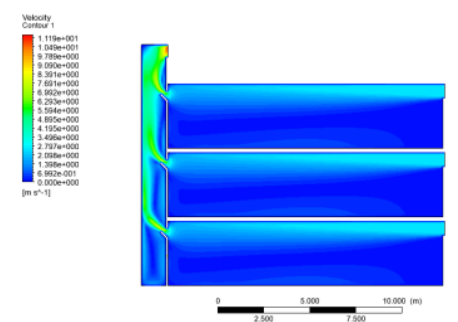

$\dot{L}$

Figure 11: Velocity field
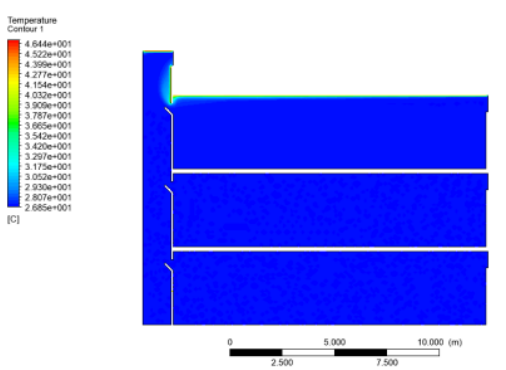

$\stackrel{\leftrightarrow}{\circ}$

Figure 12: Temperature field

Through the simulation results compared with natural ventilation solutions based on experience, it can be seen that the velocity field results are consistent with the air flow in the architecture and with the obtained temperature field, the average temperature will fluctuate between 27 and 29.5 degrees Celsius, satisfying the standard of comfort for humans.

\section{Conclusion}

The simulation results are similar to the experimental ventilation solution. However, the simulation results yield more detailed information and based on that, it is possible to improve the architecture so that the natural ventilation process is better. 
In addition, the simulation of natural ventilation by numerical method also helps to save costs. However, the paper has some limitations such as not using many different turbulent models to compare the results of which is better. The input conditions are few, only the conditions of temperature and speed have been introduced. Only evaluated for temperature and air flow velocity, not yet evaluated for air humidity

\section{List Of Acronyms}

DNS: Direct Numerical Simulation

LES: Large Eddy Simulation

RANS: Reynolds-Averaged Navier-Stokes

\section{Conflicts of Interest}

The authors declare no conflicts of interest.

\section{Acknowledgment}

We acknowledge the support of time and facilities from Ho Chi Minh City University of Technology (HCMUT) - VNU-HCM for this study.

\section{Author's Contribution}

Tich Thien Truong participate in the creation of article writing ideas, responsible for reporting results. Kim Bang Tran offer calculation algorithm, check and complete the paper.

Quoc Thien Pham perform the calculation process, write the article.

\section{References}

The Architecture of Natural Cooling, (C) 2020 Brian Ford, Rosa Schiano-Phan and Juan A. Vallejo.. An Introduction to Computaional Fluid Dynamics, (c) Pearson Education Limited 1995, 2007.

TCVN 5687:1992 on ventilation, air conditioning, heating - Design standards.

QCVN 26:2016/BYT on Microclimate - Permissible values for microclimate at work.

Ansys Fluent User's Guide.

Controlled natural air ventilation - New proposal in natural open-oriented design - Architecture Journal.

Strategies to control intelligent natural ventilation in buildings.

CFD simulation using ANSYS-FLUENT software (cfd-engineer.com). 